\title{
Growth of 2-amino-5-chlorobenzophenone single crystal by Microtube Czochralski method and its characterization
}

\author{
R GOVINDARAJ*, M MAGESH and P RAMASAMY \\ Centre for Crystal Growth, SSN College of Engineering, Chennai 603 110, Tamilnadu, India
}

MS received 20 November 2013; revised 30 March 2014

\begin{abstract}
Organic single crystals of 2-amino-5-chlorobenzophenone (2A5CB) were grown by Microtube Czochralski method using Microtube as a seed. The grown crystals were characterized by single crystal and powder X-ray diffraction. The functional groups of the grown crystal were found using Fourier transform infrared spectroscopy. The cutoff wavelength of $2 \mathrm{A5CB}$ has been identified using UV-vis-NIR studies. Thermogravimetric/differential thermal analysis (TG/DTA) has been carried out to find the thermal behaviour. 2A5CB was found to be thermally stable up to $125^{\circ} \mathrm{C}$. Powder second harmonic generation (SHG) was investigated to explore its nonlinear optical (NLO) properties. The mechanical stability of $2 \mathrm{A5CB}$ is studied by using Vickers hardness testing.
\end{abstract}

Keywords. Optical materials; crystal growth; mechanical properties; thermogravimetric analysis; X-ray diffraction; Czochralski method.

\section{Introduction}

Nonlinear optical (NLO) materials that are capable of generating the second harmonic frequency play an important role in the domain of optoelectronics and photonics. NLO materials have wide application in the field of telecommunication, optical computing and optical data storage. In the last decade, there has been tremendous progress in the development of the NLO materials having large NLO coefficients. ${ }^{1-5}$ NLO crystals should meet several requirements, such as large phase-matchable NLO coefficient, a wide optical window around the visible region, mechanical stability and a high damage threshold. ${ }^{6}$ Most of the organic materials will decompose below their melting temperature because of their thermal instability. Normally the Bridgman techniques are widely used to grow a bulk size crystal, but the impurity may be present in grown crystal and the orientation and nucleation cannot be controlled. However, in Microtube Czochralski technique the repeatability of getting single crystals is about $80 \%$ with the optimized growth parameters. Due to poor thermal conductivity of organic materials, a slight thermal fluctuation leads to more instability in solid-liquid interface in the conventional Czochralski technique whereas in Microtube Czochralski technique, Microtube is found to be improving the stability of solid/liquid interface at the initial stage of nucleation. ${ }^{7}$ From this technique we can control the nucleation and growth orientation in the Microtube by varying the parameters. 2-Amino-5-chlorobenzophenone (2A5CB) is an organic

*Author for correspondence (isrogovindaraj@gmail.com) material for $\mathrm{NLO}$ applications. $2 \mathrm{~A} 5 \mathrm{CB}$ has a molecular weight of 231.68. The molecular formula of $2 \mathrm{~A} 5 \mathrm{CB}$ is $\mathrm{C}_{13} \mathrm{H}_{10} \mathrm{ClNO}$, which belongs to the $\mathrm{C}$-face-centered monoclinic system. The difficulty of solution-grown crystals is that it is needle shaped and small sized, moreover it is not sufficient for second harmonic generation (SHG) studies. Ramesh Babu et $a l^{8}$ and Gulam Mohamed et $a l^{9}$ have reported the $2 \mathrm{~A} 5 \mathrm{CB}$ single crystal's cutoff wavelength as 440 and $390 \mathrm{~nm}$, respectively. The cutoff wavelength of Microtube Czochralskigrown 2A5CB is $456 \mathrm{~nm}$. The Microtube Czochralski was employed with the aim of growing a large size 2A5CB single crystal as well as justifying the suitability of this method for single-crystal growth. The grown crystal was subjected to single crystal and powder X-ray diffraction (XRD) studies, Fourier transform infrared spectroscopy (FTIR), UV-vis-NIR studies, thermogravimetric-differential thermal analysis (TG-DTA) and microhardness studies.

\section{Experimental}

Figure 1 shows the schematic arrangement of the Microtube Czochralski method. A $55 \mathrm{~mm}$ ID and $70 \mathrm{~mm}$ height glass crucible was placed in the Kanthal wire-wound furnace. The stainless steel (SS) Microtube was fitted with a rod which can be rotated. A SS Microtube of $6 \mu \mathrm{m}$ ID has been used for seeding the melt. The temperature profile was taken before loading the material in glass crucible. The high purity source material was directly purchased from Alfa Aesar and the material was first loaded into the glass crucible, then it was heated up to $105^{\circ} \mathrm{C}$. After attaining the visible melt, the SS Microtube was just kept above the melt surface in order 


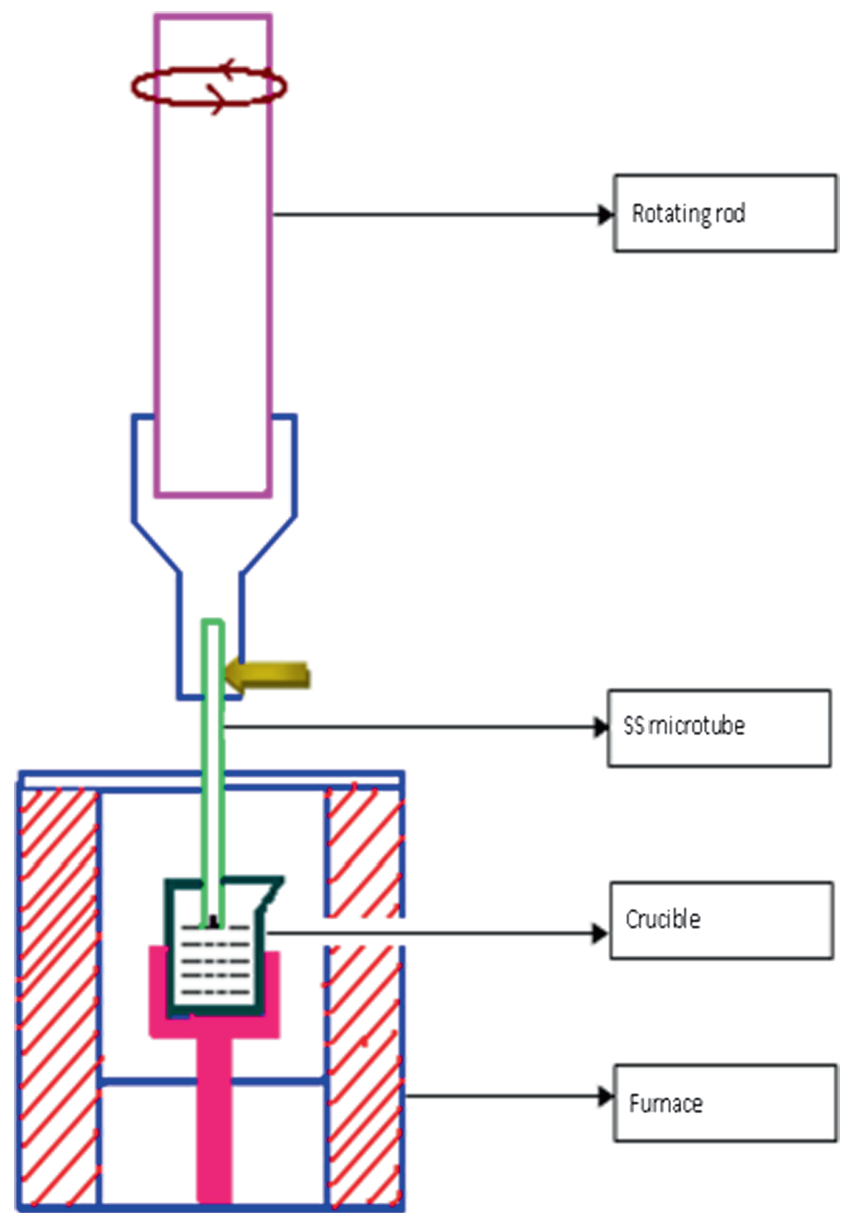

Figure 1. Microtube Czochralski method.

to attain the thermal equilibrium condition. Once the attainment of thermal equilibrium is ascertained, the microtube was brought in contact with the melt. The temperature was reduced at a predefined rate until growth occurred. A fine column of melt was rising inside the microtube because of the capillary rise. It was allowed to crystallize inside the microtube and then used as a primary seed. Melt that wets the walls of the fine capillary tube, rises to a height depending upon the tube radius and the surface tension of the melt. The pulling rate of the microtube and growing rate of the crystal are carefully optimized in order to get perfect crystal. During the initial growth period the control parameters, such as temperature and pulling rate, were carefully monitored. The resulting crystal diameter depends on the pulling rate and temperature gradient. High pulling rate with a small temperature gradient yields a smaller diameter crystal, whereas large gradient and low pulling rate results in a thicker sample. Once the growth run is completed, the system temperature is reduced at a predetermined cooling rate. ${ }^{7,10}$ The optimized experimental parameters in this present investigation for the growth of $2 \mathrm{~A} 5 \mathrm{CB}$ are as follows: pulling rate $1-2 \mathrm{~mm} \mathrm{~h}^{-1}$; seed rotation rate 8 r.p.m.; cooling rate $1^{\circ} \mathrm{Ch}^{-1}$ and the axial thermal gradient $8^{\circ} \mathrm{C} \mathrm{cm}^{-1}$. Figure 2 shows the grown crystal of $2 \mathrm{~A} 5 \mathrm{CB}$.

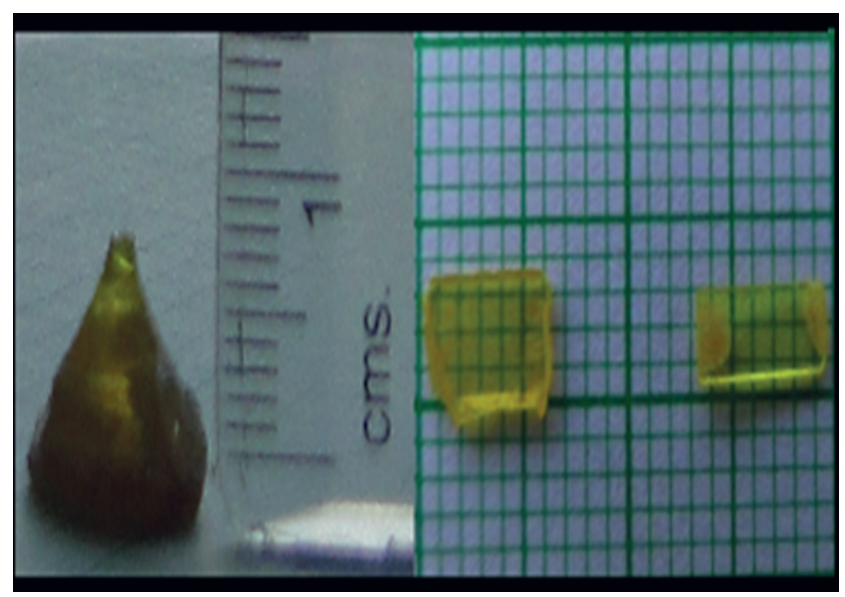

Figure 2. 2-Amino-5-chlorobenzophenone single crystal.

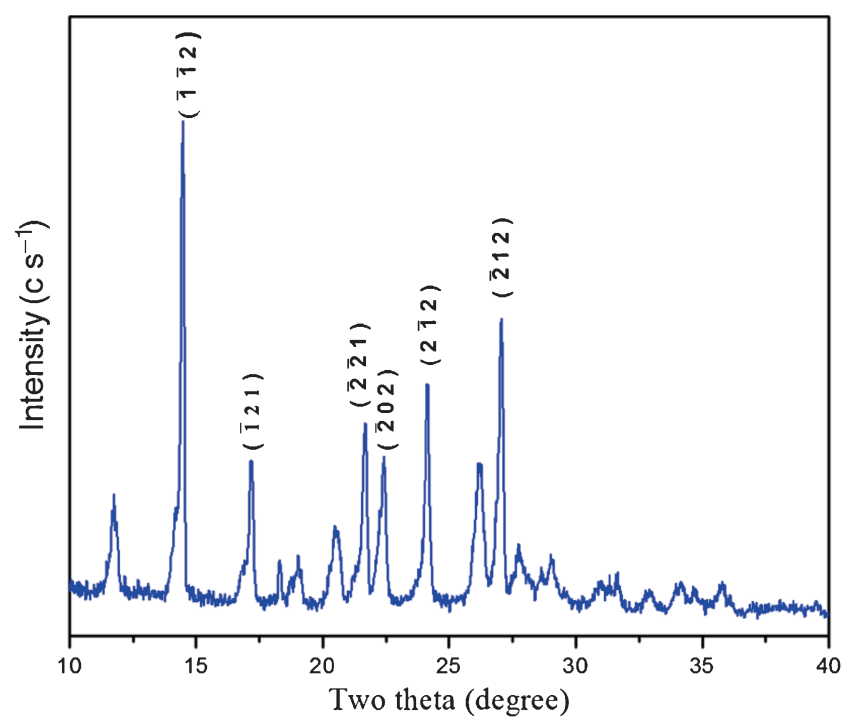

Figure 3. Powder XRD pattern of 2A5CB.

\section{Characterization studies}

\subsection{Single-crystal XRD analysis}

From the single-crystal XRD measurement, it is found that the grown crystal belongs to the $\mathrm{C}$-face-centered monoclinic system. The determined lattice parameters are $a=9.75 \AA$, $b=18.99 \AA, c=24.81 \AA$ and $\alpha=\gamma=90^{\circ}, \beta=95.78^{\circ}$ and volume $=4569.29 \AA^{3}$.

\subsection{Powder XRD}

Powder XRD studies were carried out using $\mathrm{CuK} \alpha$ $(1.54058 \AA)$ radiation, scanning angle ranging from $10^{\circ}$ to $40^{\circ}$ at a scan rate $1^{\circ} \mathrm{min}^{-1}$ to study the phase purity of the grown crystal. The XRD pattern of $2 \mathrm{~A} 5 \mathrm{CB}$ is shown in figure 3 . The obtained peaks are indexed. The sharp peaks show the crystalline nature of the sample. 


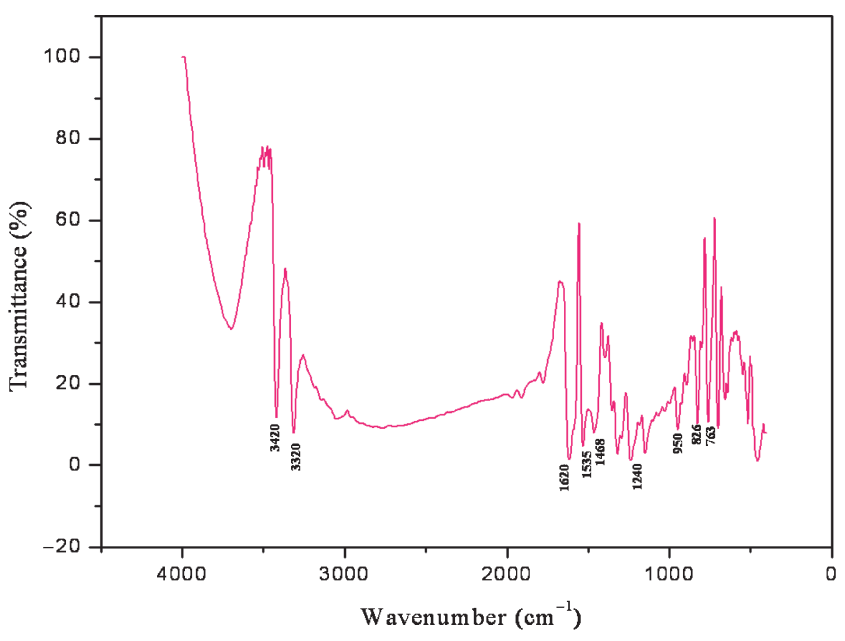

Figure 4. FT-IR spectrum of 2A5CB.

\subsection{FT-IR analysis}

Figure 4 shows the FT-IR spectrum of 2A5CB. The spectrum was recorded in the range of $4000-400 \mathrm{~cm}^{-1}$. The absorption at 3420 and $3320 \mathrm{~cm}^{-1}$ is due to $\mathrm{N}-\mathrm{H}$ stretching vibration. The $\mathrm{N}-\mathrm{H}$ group normally gives rise to one band and $\mathrm{NH}_{2}$ group gives two bands, such as symmetrical and antisymmetrical stretching. The absorption peak at $1620 \mathrm{~cm}^{-1}$ corresponds to $\mathrm{C}=\mathrm{O}$ stretching vibration of the carbonyl group. The presence of aromatic amines stretching is confirmed at $1240 \mathrm{~cm}^{-1}$. The aromatic ring of $\mathrm{C}=\mathrm{C}$ is revealed at 1468 and $1535 \mathrm{~cm}^{-1}$. The peaks at 828 and $950 \mathrm{~cm}^{-1}$ are due to $\mathrm{C}=\mathrm{C}-\mathrm{H}$ out of plane. The peak at $763 \mathrm{~cm}^{-1}$ corresponds to $\mathrm{C}-\mathrm{Cl}$ stretching vibration.

\section{$3.4 U V-v i s-N I R$ studies}

The grown 2A5CB single crystal was cut and polished, and the good transparent crystal with $3 \mathrm{~mm}$ thickness was used for UV-vis-NIR studies. These studies were carried out without any antireflection coatings. The recorded spectrum is shown in figure 5. It is observed that the grown 2A5CB single crystal has the transparency of around 50\% and the cutoff wavelength is around $456 \mathrm{~nm}$, whereas solution-grown crystal gives higher transmittance. The lower transmittance is due to the dislocations present in the melt-grown crystal.

\subsection{Thermal studies}

The results obtained from TG and DTA are shown in figure 6. The TGA and DTA traces of $2 \mathrm{~A} 5 \mathrm{CB}$ were recorded in the nitrogen atmosphere at a scanning rate of $10^{\circ} \mathrm{C} \mathrm{min}^{-1}$ from room temperature to $250^{\circ} \mathrm{C}$. The grown $2 \mathrm{~A} 5 \mathrm{CB}$ crystal was stable upto $125^{\circ} \mathrm{C}$. As shown in figure 6, a sharp endothermic peak was observed at $101.5^{\circ} \mathrm{C}$, which is assigned as the melting point. A sharp peak indicates the good degree of crystallinity of the sample. In the TGA analysis, there is a gradual and continuous weight loss beyond $125^{\circ} \mathrm{C}$. From

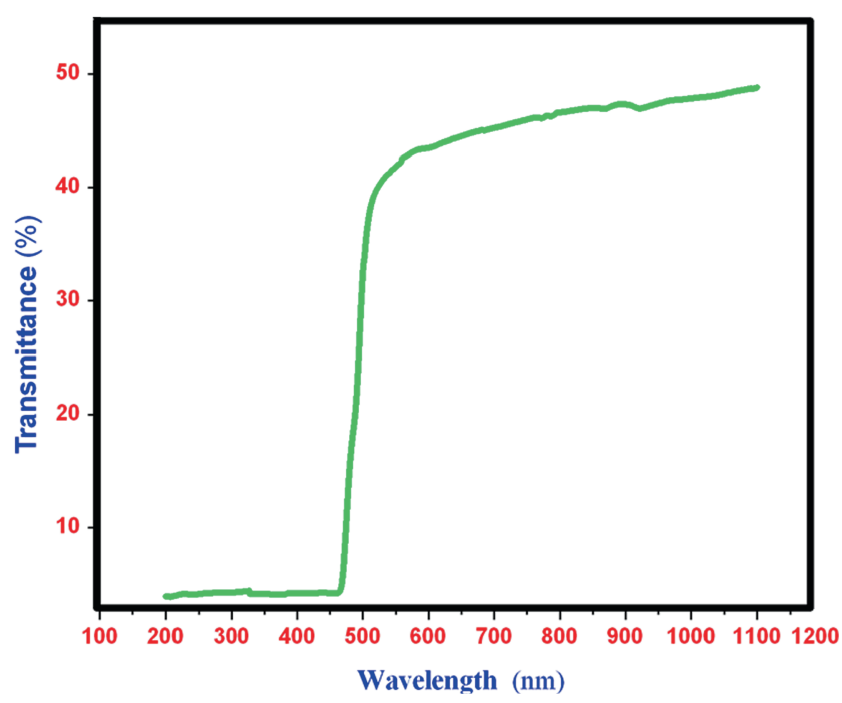

Figure 5. UV-vis-NIR spectrum of $2 \mathrm{~A} 5 \mathrm{CB}$.

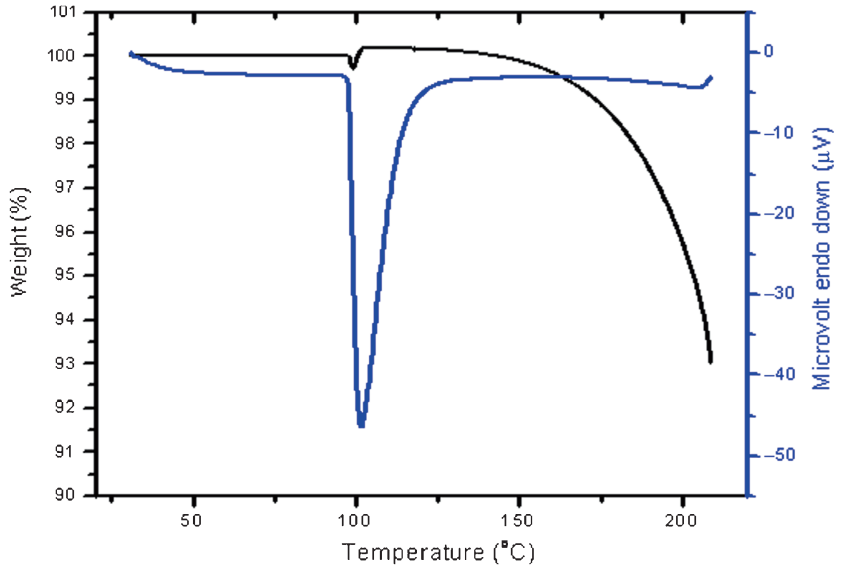

Figure 6. TG-DTA curve of 2A5CB.

these results it is concluded that there is no phase change observed in the crystal.

\subsection{Microhardness studies}

Information about mechanical hardness of NLO crystals is important not only from the device point of view, but also due to the close correlation between mechanical hardness, bond strength, laser damage, etc. ${ }^{1-13}$ Figure 7 shows the load dependence of hardness of 2A5CB. From the plot it is clear that Vickers hardness number (VHN) increases with load. This indicates the reverse indentation size effect (ISE). The measurement performed beyond a load of $100 \mathrm{~g}$ resulted in some cracks. The hardness behaviour of solution-grown crystal is found to be opposite to hardness behaviour of melt-grown crystal. The defects due to solution inclusion (in solution-grown crystals) reduce the hardness. The defects in melt-grown crystal increase the hardness as they cause resistance to lattice slipping. 


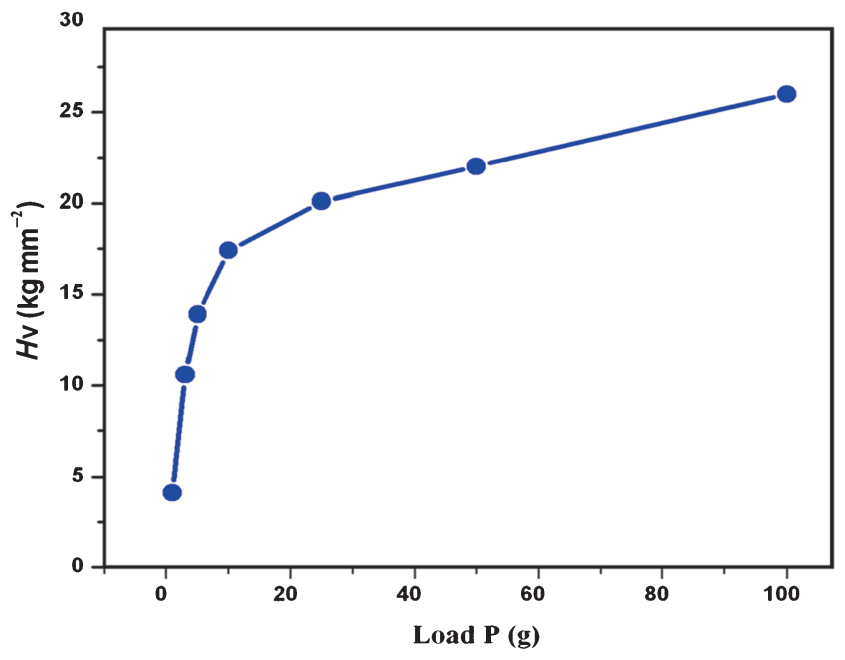

Figure 7. Vickers microhardness test of $2 \mathrm{~A} 5 \mathrm{CB}$.

\subsection{SHG efficiency}

The Kurtz-Perry powder technique is an extremely valuable tool for SHG. The ground powder was packed in a triangular cell and kept in a cell holder. A Q-switched Nd-YAG laser beam of wavelength $1064 \mathrm{~nm}$ with the energy of the laser pulse around $4.7 \mathrm{~mJ}$ was allowed to strike the sample cell normally. The beam emerging through the sample was focused on to a Czerny-tuner monochromator using a pair of lenses. The detection was carried out using a Hamamatsu R-928 photomultiplier tube. The signals were captured with an Agilent infiniium digital storage oscilloscope interfaced to a computer. The monochromator is set at $532 \mathrm{~nm}$. NLO signal is captured by the oscilloscope through the photomultiplier tube. The intensity of the green emission is estimated to be as 0.3 times that of the reference KDP sample.

\section{Conclusion}

2A5CB single crystal was successfully grown by the Microtube Czochralski method. The grown crystal was characterized by single-crystal XRD, FT-IR and PXRD. The grown crystal belongs to C-face-centered monoclinic system, which is confirmed with single-crystal XRD studies. The material was stable up to $125^{\circ} \mathrm{C}$ and it shows there is no phase change present in the melt-grown crystal. The UV-vis-NIR study shows the cutoff wavelength as $456 \mathrm{~nm}$ and its transmittance is $50 \%$ in the vis-NIR region. This is moderately good for melt-grown crystals. The SHG efficiency of 2A5CB is 0.3 times that of KDP. Microhardness studies indicate the occurrence of reverse indentation size effect in the grown crystal. Finally, we claim that the Microtube Czochralski is suitable technique for growing a large single crystal without a seed. Due to its versatility and repeatability of getting bulk single crystals, this method can also be extended to grow other newer materials.

\section{Acknowledgements}

We are thankful to Dr R Gopalakrishnan, Department of Physics, Anna University, for providing the hardness facility, as well as IIT Madras and IISc Bangalore for giving the single-crystal X-ray diffraction and the SHG facility, respectively.

\section{References}

1. Rajesh N P, Kannan V, Santhanaragavan P, Ramasamy P and Lan C W 2002 J. Mater. Lett. 52326

2. Mallik T and Kar T 2005 Cryst. Res. Technol. 40778

3. Ambujam K, Rajarajan K, Selvakumar S, Ginson I V, Joseph P and Sagayaraj P 2006 J. Cryst. Growth 286440

4. Kannan V, Bairava Ganesh R, Sathyalakshmi R, Rajesh N P and Ramasamy P 2006 Cryst. Res. Technol. 41678

5. Madhavan J, Aruna S, Prabha K, Packium Julius K, Ginon P Joseph, Selvakumar S and Sagayaraj P 2006 J. Cryst. Growth 293409

6. Chemla D S and Zyss J 1987 Nonlinear optical properties of organic molecules and crystals (New York: Academic Press) Vols. 1 and 2

7. Arivanandhan M, Ramamoorthy K, Sankaranarayanan K, Sanjeeviraja C and Ramasamy P 2005 Opt. Mater. 271864

8. Ramesh Babu R, Vijayan N, Gunasekaran M, Gopalakrishnan $\mathrm{R}$ and Ramasamy P 2004 J. Cryst. Growth 265290

9. Gulam Mohamad M, Rajarajan K, Mani G, Vimalan M, Prabha K, Madhavan J and Sagayaraj P 2007 J. Cryst. Growth 300409

10. Sankaranarayanan K and Ramasamy P 1998 J. Cryst. Growth 193252

11. Li K Y and Xue D F 2009 Chin. Sci. Bull. 54131

12. Li K Y and Xue D F 2010 Phys. Scr. T139 014073

13. Li K, Wang X, Zhang F and Xue D 2008 Phys. Rev. Lett. 100 235504 